\title{
CARDIOMEGALIA GLYCOGENICA CIRCUMSCRIPTA
}

\author{
BY
}

\author{
S. VAN CREVELD, M.D. and H. M. VAN DER LINDE, M.D.
}

(From the Propaedeutic Clinic and the Pathological Laboratory of the University of Amsterdam)

In 1932 Pompe first drew attention to hypertrophy of the heart caused by excessive accumulation of glycogen in the heart muscle. Since then experience has shown that such an accumulation of glycogen could indeed explain many of the cases of hypertrophy of the heart in infants, which formerly would have been labelled as cases of idiopathic hypertrophy of the heart, for the enlargement could not be explained by a defect of valve or septum. Later, however, it appeared that there were cases of so-called idiopathic hypertrophy of the heart in infants, without accumulation of glycogen and not showing the typical structure of the cardiac muscle fibres found in cases of cardiomegalia glycogenica (Debré, 1935 ; Mutgeert, 1937 ; Mansens, 1937). There is still a third form of congenital hypertrophy of the heart which clinically appears to be an idiopathic or an essential one. In these cases, however, pathological and microscopical examination prove the existence of defects of the heart muscle, with or without corresponding abnormalities in the coronary vessels (Kugel and Stoloff, 1933). This form is sometimes called the secondary or pseudoidiopathic form of hypertrophy of the heart.

The question if a sharp separation should be maintained in future between these different forms of congenital hypertrophy of the heart cannot at the present time be answered with certainty. There is some evidence that a relation between the different forms does exist. There is the fact that in cases of pseudoidiopathic hypertrophy of the heart localized changes in the heart muscle have been found of the same nature as found diffusely spread in the heart muscle in cases of cardiomegalia glycogenica. One observation of such a case has recently been published in the literature; some time ago we observed an analogous case.

Carrington and Krumbhaar in 1924, under the title 'So-called Idiopathic Cardiac Hypertrophy in Infancy,' described a case of a girl ten months old. Clinically there was a large heart and a palpable liver and spleen. At the autopsy a greatly enlarged heart was found. The origin of the left coronary artery was abnormal ; it originated from the pulmonary artery. Though on macroscopical examination the different parts of the heart had a homologous aspect, the microscopical investigation of the myocardium showed the existence of areas of large waving muscle fibres with 'vacuolar degeneration.' In some 
places a slight diffuse fibrosis was found. Staining for glycogen or fat was not performed at that time. After publication of the abnormalities found in cases of the cardiomegalic type of glycogen disease, this diagnosis was considered in the case reported by Carrington and Krumbhaar. In small pieces of the heart muscle of this case, which had been kept enclosed in paraffin for thirteen years, Finkelstein (1936) was able to demonstrate glycogen in the vacuolized areas by means of the carmine stain. Finkelstein was of the opinion therefore that it was justifiable to say that in the case described by Carrington and Krumbhaar there existed localized changes in the heart muscle, which histologically and histochemically showed resemblance to the findings in glycogen disease. Histologically the structure differed from that found in cases of cardiac rhabdomyomas, which also contain much glycogen. In order to differentiate this case from the more diffuse changes found in the myocardium in cases of the cardiomegalic type of glycogen disease, Finkelstein called the condition 'cardiomegalia glycogenica circumscripta.'

\section{Case record}

Baby A., a girl, was taken into the Babies Department of the Propaedeutic Clinic of the Wilhelmina-Hospital (director: Prof. I. Snapper) when five months old. She was the first child of healthy parents, born spontaneously and at full term. She had been under regular supervision as regards feeding and general condition. Until some days before admission to the hospital the child had not shown any peculiarity, except that during the previous two months she had gained little weight. Five days before admission she had fallen ill with dyspnoea and laboured breathing; she coughed and whimpered, vomited, perspired abundantly, showed anorexia and a pale complexion, but no fever.

In hospital the child appeared to be ill ; she whimpered and had rapid, shallow respiration with active dilatation of the alae nasi. There was no cyanosis and the nutritional state was moderate. The right cheek and upper lip showed remnants of an angioma treated by means of radium. There was marked inspiratory recession of the soft parts of the chest. The heart was greatly enlarged to the right and to the left with loud sounds and a soft systolic murmur, especially at the apex of the heart and just below the sternum. The lungs showed no abnormality on percussion or auscultation. The spleen was not palpable ; the liver was enlarged and palpable three fingers'-breadths below the costal arch, and to the left it reached two fingers'-breadths past the middle line. The back of the foot on each side showed slight oedema. The reflexes were normal. The urine contained albumin and urobilin. The sediment contained an occasional erythrocyte and some leucocytes. The blood picture was normal. An x-ray investigation of the thorax (fig. 1) showed a greatly enlarged heart, to the left as well as to the right ; in both lungs there were a large number of darker areas (congestion ?). Electrocardiogram (Dr. Formyne) : P III negative, rather deep S I and S II ; T II and T III negative. The fundus oculi showed no abnormality. X-ray photographs of the skull were normal.

The possibility had to be considered of the existence of a glycogen heart as this organ was greatly enlarged without definite evidence of a defect of the valves or other congenital abnormality. Moreover, until admission to hospital there had been no symptoms, a fact which has frequently been observed in cases of glycogen heart. Furthermore, in this stage of the disease the weak murmur did not exclude the possibility of a glycogen heart. The development of the clinical symptoms indicated that the enlarged liver was probably a congested liver.

Certain investigations were performed in order to find other signs as usually found in glycogen-liver (van Creveld, 1928, 1934). The urine on repeated 
examination never contained acetone or sugar, and in the blood in the fasting state acetone and $\beta$-oxybutyric acid were absent. The fasting blood-sugar was 0.075 per cent. (Hagedorn-Jensen). The glycogen content of the blood in fasting condition was increased $(34.5 \mathrm{mgm}$. per $100 \mathrm{cc}$.). After injection of $0.25 \mathrm{cc}$. of adrenalin 1/1000 the blood-sugar content had risen after one hour from 0.075 per cent. to 0.105 per cent.

These observations gave scant evidence in favour of the diagnosis of the cardiomegalic type of glycogen disease. The electrocardiogram was highly suggestive of myocardial damage. The possibility of confirming the diagnosis

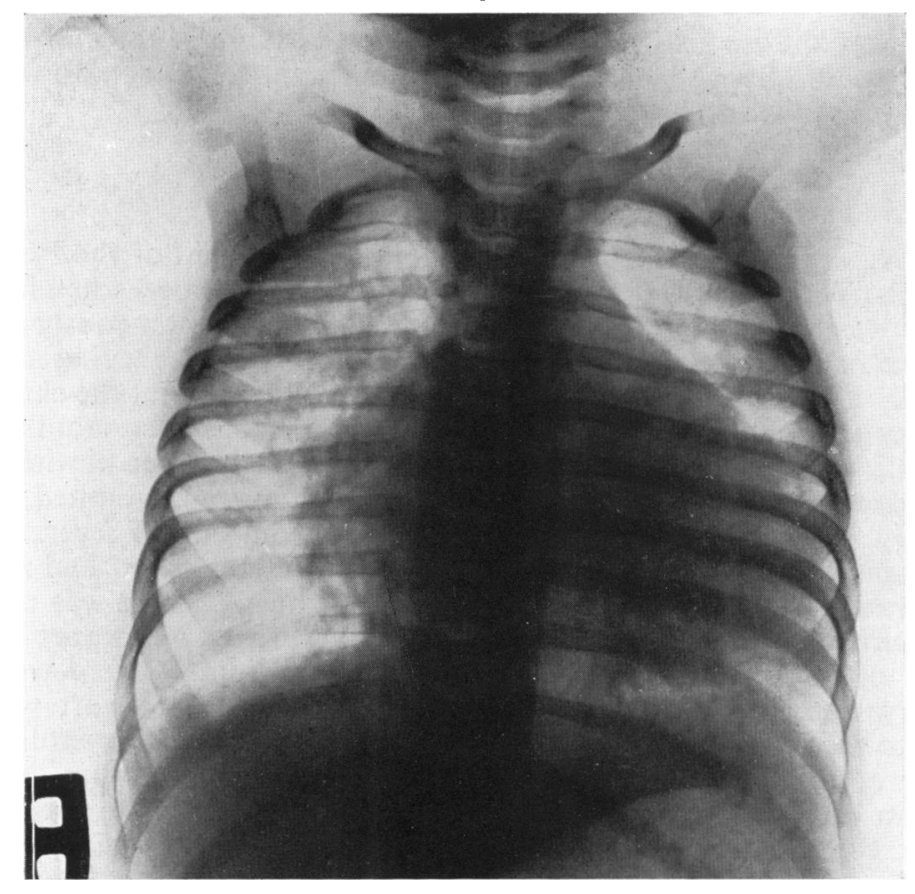

FIG. 1.-X-ray film of the thorax showing the proportions of the heart.

of glycogen disease by examination of some striated muscle fibres obtained by biopsy (Humphreys and Kato, 1934) could not be considered in view of the serious general condition of the patient.

During the nine days of the child's stay in the hospital the oedema of the lower extremities increased; cyanosis and elevation of the temperature remained absent. The von Pirquet and Wassermann reactions were negative. The condition of the heart remained the same, the liver decreased somewhat in size. On the tenth day after admission to hospital the child died suddenly.

At necropsy there was a marked degree of hypertrophy of the heart with a large patent foramen ovale, dilatation of the pulmonary artery-system and peculiar focal lesions in the heart muscle itself, with thickening of endocardium and epicardium. The liver was greatly enlarged, of firm consistency and contained much blood. The lungs were very congested. The kidneys also showed signs of congestion. The adrenals were normal. 
It appeared that the diagnosis ' cardiomegalia glycogenica,' already unlikely on account of these findings, had to be rejected when some sections of the heart muscle examined immediately after the autopsy showed no trace of the histological abnormalities characteristic of the 'glycogen heart.' The quantitative determination of the glycogen in a small piece of the heart muscle gave a result of 0.5 per cent., a value which did not resemble the high values found in cases of 'glycogen heart,' but which still was higher than the values usually found at that age in examining the heart a considerable time after death. At this time it was not appreciated how these findings could be fitted together.

The combination of lesions found on macroscopical examination of the

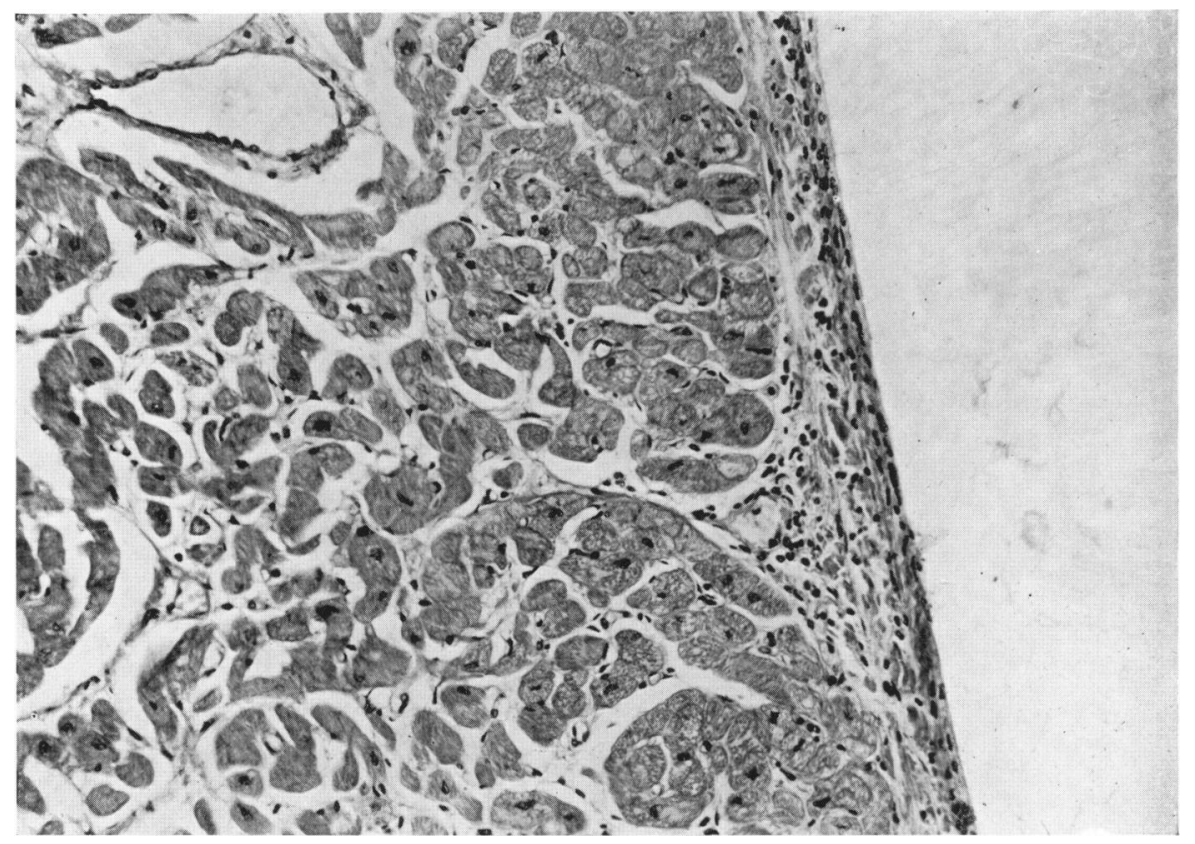

FIG. 2.-Muscle-fibres situated sub-epicardially, showing swelling, granulation and vacuolization of the plasma.

heart and of the pulmonary artery, although rather unusual, has been more frequently observed as a congenital malformation (Joules, 1934 ; d'Aunoy and von Haam, 1934 ; Okkels and Therkelsen, 1932). One of us, a short time ago, found this combination of lesions at the autopsy of an adult man. But the present case differed in many respects on macroscopic examination from the reported cases ; the hypertrophy of the heart existed on both sides. Further, in this case the heart muscle showed extensive focal lesions, whereas in the reported cases there was at most a slight fibrosis.

Microscopic ExAmination. No abnormality of thymus, pancreas, adrenals, spleen and kidneys was found. Best's carmine stains were negative in these organs. The striated (diaphragm) and the smooth muscle tissues (pyloric muscle, oesophagus and duodenum) showed no abnormalities ; in 
particular, the stains for glycogen also proved to be negative here. The lungs showed chronic congestion with some dilatation of the branches of the pulmonary artery, even quite near the pleura. The liver showed congestion, with scattered small haemorrhages. Both near the central vein, and more peripherally in the lobules there were a number of small areas in which the liver cells were larger and paler than normal, with sharp outlines of the cells and a fine granular plasma without vacuoles. Stains after Best proved the existence of glycogen in these cells.

In the examination of the heart, epicardium and coronary arteries were normal ; the endocardium did not show changes of any importance. In the myocardium there were extensive scattered areas, which were abnormal. In the first place these areas, chiefly under the endocardium, had a structure which

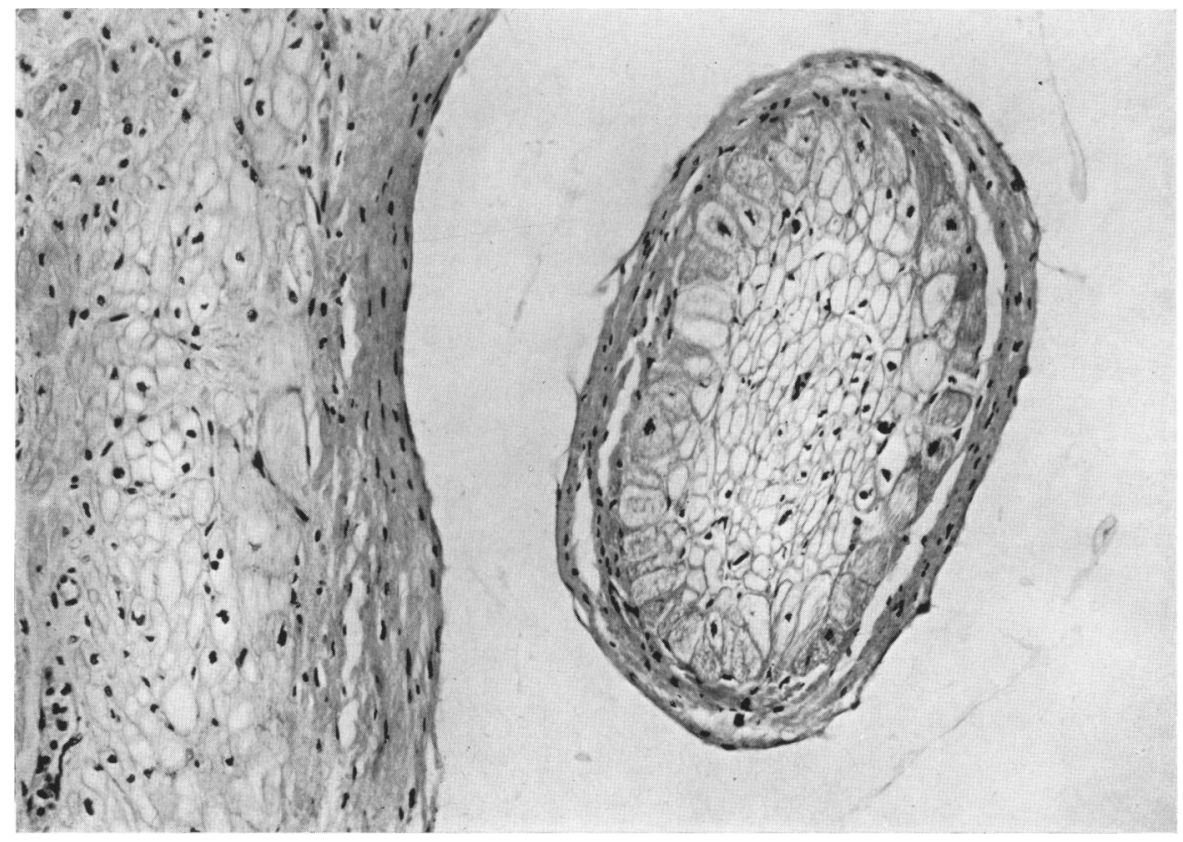

FIG. 3.-Structure typical of the heart-muscle fibres in glycogen heart, in a papillary muscle and in the sub-endocardial part of the myocardium.

is almost characteristic of glycogen degeneration of the muscle fibres : namely, large, long, thick fibres with clear, vacuolar protoplasm. The nuclei lay in the centre, sometimes flattened against the sarcolemma. The borders of these fibres were often distinctly striated. After staining for glycogen only some fibres showed typical, fine-granular drops of glycogen, some around the central nucleus and others more peripherally at the ends of the fibres. The intracellular glycogen granules, however, are present only in those areas where a real glycogen structure is found. Thus any contamination can be excluded (fig. 2, 3 and 4).

The second group of small fields in the myocardium, showing an abnormal structure, consisted of necrotic spots (fig. 5) and muscle fibres with characteristic coarse, globular fatty degeneration, arranged in small groups like forked lightning. Here the glycogen structure is completely absent. There are, however, some fibres which are somewhat swollen, with clear outlines and 


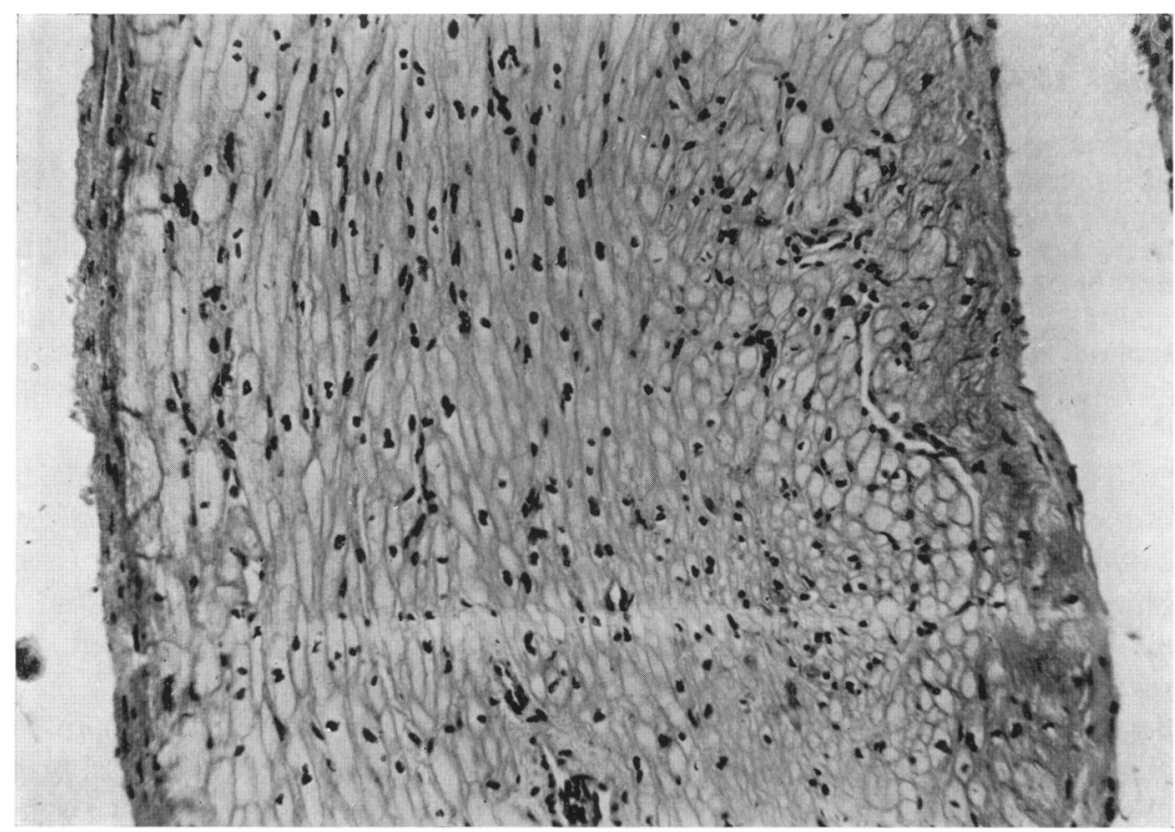

FIG. 4.-Typical vacuolized structure in a papillary muscle ; distinct striation is still present.

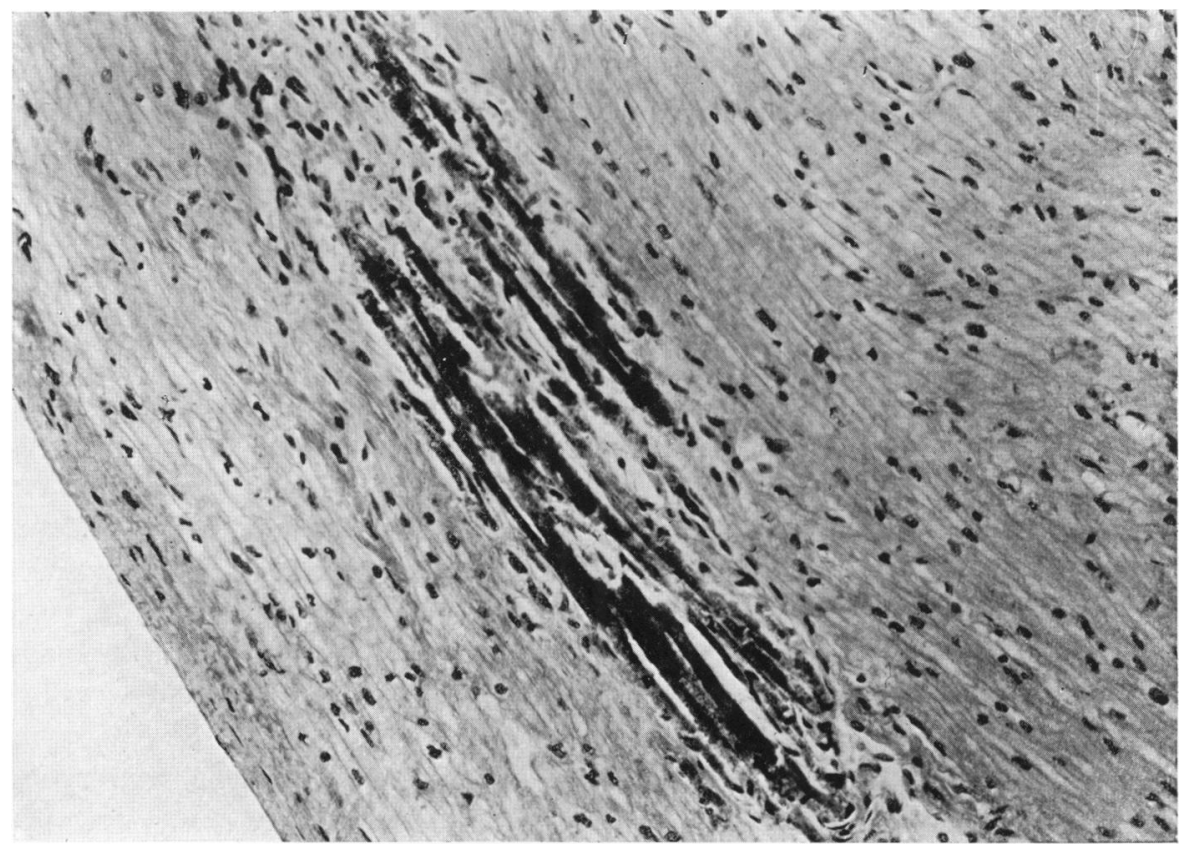

Fig. 5.-Typical necrotic area in the myocardium. 
a pale, distinctly granular plasma, with vacuoles (similar to those observed in the case of an adult, in whom there had existed for three hours an obstruction of the big branch of the left coronary artery). Further the myocardium shows small fields of atrophic muscle fibres, where each fibre is surrounded by thickened interstitial tissue ; and a number of small places, made up of thick ' Scholl 'like muscle fibres, which in the haematoxylin-sections take an intensely blue colour and in the van Gieson sections an intensely yellow one. Next to these there are, moreover, small fields (a less distinct passing from one kind into another ?) where the fibres show a coarse structure with absent or very pale nuclei. These fibres take an intensely red colour in the haematoxylin-eosin sections and where the cross-striation is lacking. Finally, in a still more advanced stage, there are small groups of fibres with granulation and a bluecolour in the haematoxylin-eosin-sections.

To summarize the foregoing it may be said that here is a case of hypertrophy of the heart, founded or not on a congenital deformity (the large patent foramen ovale and a dilatation of the whole extent of the pulmonary artery). Combined with these abnormalities there are congenital and (or) acquired abnormalities in the myocardium, namely partial accumulation of glycogen and regressive changes (increase of connective tissue, necrosis, especially fatty degeneration) as a consequence of older or more recent disturbances in the circulation. On histological examination the places where accumulation of glycogen was found showed a striking resemblance to those found by Pompe and others in cases of cardiomegalia glycogenica. In the liver there were small fields with a structure typical of glycogen accumulation (von Gierke, 1929).

\section{Discussion}

The following possibilities in explaining the partial accumulation of glycogen as found here have to be considered :

1. The condition results from an unusually dominant development of the conducting system, in which, as is known, at any rate during the embryonic period, analogous structures exist. Most of the abnormalities are, however, not in agreement with this conception. Furthermore, in the case of cardiomegalia glycogenica circumscripta, described by Finkelstein and cited in the beginning of this report, the glycogen fields were not lying especially subendocardially, as in this case.

2. This might be a persistence of the embryonic condition, in which the embryonic structure of the myocardium has for unknown reasons been maintained, especially in the subendocardial area. This would infer that the cases of glycogen heart of Pompe and others would have to be considered-as formerly had been supposed (Pompe, 1936) - as a congenital condition, in which the whole myocardium had remained in an embryonic condition. Looked upon from this point of view, the name glycogen degeneration would be incorrect ; for then there should not be any question of a change of fibres which originally had had a proper structure, but of a persistence of the embryonic structure of the fibres. Why the fibres retain more or less their embryonic state is a question which cannot be answered; perhaps there exists a relation with some disturbance in the circulation, for in this case there were also many regressive changes which had been caused by a poor circulation. In Finkel- 
stein's case also disturbances in the circulation were found (abnormal origin of the coronary artery); however, the regressive symptoms were few (only slight fibrosis). [In the fifth case of glycogen heart discussed by Pompe (1936) (case of Frank-Dykstra) there was a circumscribed accumulation of glycogen and at the same time a defect of the heart (aneurysm of the widely dilated ductus Botalli).] The significance of disturbances in the circulation for the occurrence of glycogen in the heart might be that the proper combustion of the glycogen in embryonic structures, which are rich in glycogen, was disturbed by a poor circulation. At any rate, the occurrence of this accumulation of glycogenalthough partial-in a heart which, as in this case, also showed necrosis, fatty degeneration and fibrosis remains very unusual.

3. According to the conception just outlined the circumscribed accumulation of glycogen should represent a further stage in the development of the myocardium than the diffuse glycogen heart. However, this diffuse glycogen accumulation might also be a manifestation of a disturbance in metabolism, and then the circumscribed accumulation of glycogen may be considered to be a stage of recovery. If this hypothesis is correct, the existence of some differences between clinical and anatomical findings in both conditions is quite rational.

All these possibilities can only be solved after extensive research and after further experience of the condition. It is highly probable, nevertheless, that circumscribed accumulation of glycogen with the typical histological appearances in congenitally hypertrophied hearts may prove to be more frequent than at present realized.

\section{Summary}

A description is given of a case of cardiac hypertrophy resembling the pseudo-idiopathic type with partial accumulation of glycogen in the heart muscle. On histological examination the areas in which accumulation of glycogen was found showed a striking resemblance to those found by Pompe and others in cases of cardiomegalia glycogenica. In the liver also small areas with a structure typical of glycogen disease were present. Various possible explanations of this 'cardiomegalia glycogenica circumscripta' are discussed.

\section{REFERENCES}

d'Aunoy, R., and Haam, E. von (1934). J. Path. and Bact., 39, 1.

Carrington, G. L., and Krumbhaar, E. B. (1924). Amer. J. Dis. Child., 27, 449.

Creveld, S. van (1928). Nederl. Maandschr. v. Geneesk., 15, 349.

(1934). Arch. Dis. Childh., 9, 9.

Debré, R., Marie, J., and Bernard, J. (1935). Bull. Soc. méd. Hôp. de Paris, 995.

Finkelstein, L. E. (1936). Amer. J. med. Sci., 191, 415.

Gierke, E. von (1929). Beitr. path. Anat., 82, 497.

Humphreys, E. M., and Kato, K. (1934). Amer. J. Path., 10, 589.

Joules, H. (1934). Lancet, 2, 1338.

Kugel, M. A., and Stoloff, E. G. (1933). Amer. J. Dis. Child., 45, 828.

Mansens, B. J. (1937). Maandschr. v. Kindergeneesk., 6, 246.

Mutgeert, B. L. (1937). Loc. cit., 233.

Okkels, H., and Therkelsen, F. (1932). Acta scand. path. microbiol., 9, 214.

Pompe, J. C. (1932). Ned. Tÿdschr. v. Geneesk., 76, 304.

— (1936). Dissertation, Amsterdam. 